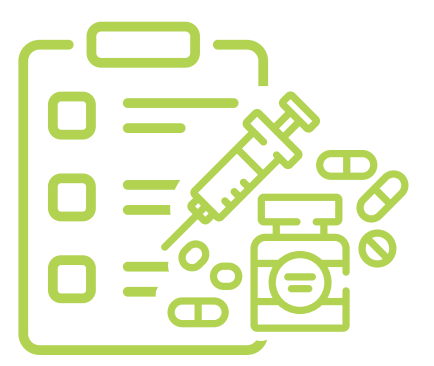

\title{
CORRELAÇÃO ENTRE A NOTIFICAÇÃO DE SÍFILIS, DISPONIBILIDADE DE PENICILINA E TESTE RÁPIDO: Uma análise a partir do sistema Retratos da Atenção Primária à Saúde
}

\begin{abstract}
Angélica Espinosa Barbosa Miranda
Doutora em Saúde Pública pela Fundação Oswaldo Cruz, Mestre em Doenças Infecciosas pela Universidade Federal do Espírito Santo e graduada em Medicina pela Escola de Medicina da Santa Casa de Misericórdia de Vitória. Coordenadora de Vigilância das Infecções Sexualmente Transmissíveis do Departamento de Condições Crônicas e IST, Secretaria de Vigilância em Saúde, Ministério da Saúde.

E-mail: angelica.miranda@aids.gov.br
\end{abstract}

\section{Gustavo Fontoura de Souza}

Doutor em Engenharia Elétrica e da Computação e Mestre em Engenharia Elétrica pela Universidade Federal do Rio Grande do Norte (UFRN). Graduação em Estatística. Professor do IFRN, Campus Parnamirim e Pesquisador do Núcleo Avançado de Inovação Tecnológica (NAVI/IFRN). Pesquisador do Laboratório de Inovação Tecnológica em Saúde (LAIS/UFRN).

E-mail: gustavo.fontoura@ifrn.edu.br

\section{Janaína Luana Rodrigues da Silva}

Doutoranda em Estudos Contemporâneos na Universidade de Coimbra, Especialista em Informática na Saúde pela Universidade Federal do Rio Grande do Norte - UFRN e Pesquisadora do Laboratório de Inovação Tecnológica em Saúde (LAIS/HUOL/UFRN) e do Núcleo Avançado de Inovação Tecnológica (NAVI/IFRN).

E-mail: Janaina.rodrigues@navi.ifrn.edu.br

\section{João Paulo Queiroz dos Santos}

Doutor e Mestre em Engenharia Elétrica e de Computação pela Universidade Federal do Rio Grande do Norte. Coordenador do Núcleo Avançado de Inovação Tecnológica (NAVI/IFRN). Pesquisador do Laboratório de Inovação Tecnológica em Saúde (LAIS/UFRN).

E-mail: joao.queiroz@ifrn.edu.br.

\section{Pablo Holanda Cardoso}

Doutorando em Engenharia Elétrica e de Computação, Mestre em Gestão e Inovação em Saúde e Graduado em Engenharia de Computação e Automação Industrial pela Universidade Federal do Rio Grande do Norte (UFRN). Pesquisador do Núcleo Avançado de Inovação Tecnológica (NAVI/IFRN).

Pesquisador do Laboratório de Inovação Tecnológica em Saúde (LAIS/UFRN).

E-mail: pablo.holanda@navi.ifrn.edu.br. 
Graduada em Tecnologia da Informação e graduanda em Ciência da Computação pela Universidade Federal do Rio Grande do Norte (UFRN). Técnica em Informática pelo Instituto Federal do Rio Grande do Norte (IFRN). Pesquisadora Núcleo Avançado de Inovação Tecnológica (NAVI/IFRN). Pesquisadora do Laboratório de Inovação Tecnológica em Saúde (LAIS).

E-mail: mayra.azevedo@navi.ifrn.edu.br.

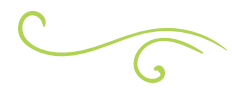

\section{RESUMO}

A sífilis é um problema de saúde pública que possui uma proporção mundial e, no Brasil, existem diversas estratégias elaboradas pelo governo para acompanhar e combater essa infecção. Dentre as iniciativas que ajudam nesta causa, encontra-se - Programa de Melhoria do Acesso e da Qualidade da Atenção Básica (PMAQ-AB). Este artigo busca, por meio dos dados obtidos do terceiro ciclo do programa, estudar a relação entre as notificações de casos de sífilis com a disponibilidade de testes rápidos, exames laboratoriais e medicamentos. Analisando os dados do Sinan e do sistema Retratos da Atenção Primária à Saúde, verificou-se que o fornecimento de testes rápidos e medicamentos, em algumas regiões, não acompanha o alto número de casos de sífilis notificados, indicando escassez na disposição dos itens.

Palavras-chave: PMAQ-AB. Sífilis. Análise de dados. Correlação.

\section{ABSTRACT}

Syphilis is a public health problem that has widespread through the world. In Brazil, the government has elaborated several strategies for monitoring and fighting this infection. Among the initiatives that contribute to this cause, there is the Program for Improving Access and Quality in Primary Care (PMAQ-AB). This article aims, through the data collected in the third cycle of the aforesaid program, to study the relationship between syphilis cases notification and the availability of penicillin, rapid tests, and exams. After analyzing data from the Sinan database and the Retratos da Atenção Primária à Saúde database, we verify that in some regions the supply of rapid tests, laboratory exams, and medicines does not match the high number of syphilis cases notified, indicating scarcity in the disposal of the necessary provisions.

KEYwords: PMAQ-AB. Syphilis. Data analysis. Correlation.

\section{INTRODUÇÃO}

A sífilis é uma Infecção Sexualmente Transmissível (IST) curável e exclusiva ao ser humano, causada pela bactéria Treponema pallidum, transmitida predominantemente por via sexual ou vertical (BRASIL, 2018). A infecção é um problema de saúde pública em escala global que foi tema da Assembleia Mundial de Saúde em 2016, na qual adotaram-se estratégias e metas para reduzir a incidência de sífilis em adultos (OMS, 2016). Seguindo a tendência mundial, no Brasil, o aumento dos casos notificados tem acelerado ao longo dos anos.

A incidência de sífilis congênita aumentou a uma taxa de 3,8 vezes no período de 2010 a 2018, atingindo a taxa de 9,0 casos por mil nascidos vivos (BRASIL, 2019). Além disso, o índice de detecção de sífilis em gestantes aumentou 6,1 vezes, elevando a taxa de 3,5 
para 21,4 casos por mil nascidos. Ademais, os casos de sífilis adquirida aumentaram de 34,1 casos por 100.000 habitantes em 2015 para 75,8 casos por 100.000 habitantes em 2018. Apesar do crescimento dos números nacionais da infecção, essa taxa de incidência se deve também a maior cobertura de testagem, assim como aprimoramento do sistema de vigilância da doença na Atenção Primária à Saúde (BRASIL, 2019).

Embora apresente números expressivos, o tratamento da sífilis adquirida (que afeta adultos e gestantes) é efetivo e de baixo custo em gestantes e seus parceiros sexuais, feito majoritariamente pela aplicação de penicilina. Já a sífilis congênita acomete recém-nascidos e, além do grande impacto social relacionado às sequelas da doença, tem um alto custo financeiro para o sistema de saúde (SORTICA, 2017).

Nesse cenário, o Ministério da Saúde elaborou diversas estratégias nacionais de combate a este agravo no Brasil. As notificações compulsórias de sífilis congênita em todo território nacional foram instituídas em 1986; a de sífilis em gestantes em 2005; por fim, sífilis adquirida em 2010. Essas notificações abrangem tanto os serviços de saúde públicos quanto os privados, gerando dados que são armazenados no Sistema de Informação de Agravos de Notificação (Sinan). Estas informações servem para o desenvolvimento de instrumentos de disseminação de informação estratégica aos gestores, auxiliando nas tomadas de decisões de políticas públicas (BRASIL, 2019).

Outra proposta relevante no enfrentamento à epidemia no país parte da Secretaria de Vigilância em Saúde, do Ministério da Saúde é o projeto de pesquisa "Pesquisa Aplicada para Integração Inteligente Orientada ao Fortalecimento das Redes de Atenção para Resposta Rápida à Sífilis"- também chamado de "Sífilis Não" que propõe uma agenda destinada a 100 municípios prioritários, que respondem a aproximadamente $65 \%$ dos casos de sífilis do Brasil. $\bigcirc$ projeto produz ações voltadas à redes de atenção primária, potencializa técnicas de vigilância da doença e elabora novos estudos operacionais (BRASIL, 2018) e assim colabora nas ações de vigilância e assistência da sífilis.

Além das informações das notificações, outro quesito que auxilia a tomada de decisão é conhecer o território onde as pessoas vivem, os serviços de saúde existentes, o fluxo de atendimentos e as características epidemiológicas e socioeconômicas locais, a fim de compreender o usuário como sujeito que tem suas concepções de saúde-doença, em sua condição social, econômica, familiar e em sua condição de gênero, principalmente quando se fala sobre uma IST como a sífilis (SORTICA, 2017).

Nesse sentido, o Programa Nacional de Melhoria do Acesso e da Qualidade da Atenção Básica (PMAQ-AB), estabelecido pelo Ministério da Saúde, conta com a plataforma Retratos da Atenção Primária à Saúde. O portal é voltado para ajudar gestores da área da saúde a monitorar a atenção primária e a população em geral a conhecer o Sistema Único de Saúde (SUS), além de permitir que pesquisadores extraiam o conhecimento que pode ser obtido através da ferramenta. Para isso, o site promove - acesso a informações colhidas durante - $3^{\circ}$ ciclo do PMAQ sobre infraestrutura, assistência e materiais referentes às unidades e equipes de saúde primária do país, bem como dados sobre os usuários do SUS acerca do atendimento recebido e de fatores demográficos e socioeconômicos do indivíduo (BRASIL, 2020).

$O$ presente artigo objetiva analisar a capacidade e o desempenho das unidades básicas de saúde no que tange ao provimento de medicamentos, testes diagnósticos e ao atendimento voltado para o controle da sífilis, bem como averiguar a eficácia do sistema para fornecer as informações necessárias para executar a tarefa proposta. Para compreender melhor a epidemia de sífilis no país, o artigo analisa a relação entre variáveis do $\mathrm{PMAQ}$ presentes 
no sistema Retratos da Atenção Primária à Saúde e os dados do Sinan. Os dados estudados são cruzados a fim de explorar se há correlação entre as notificações dos casos da sífilis e disponibilidade de penicilina e aplicações de teste rápido.

\section{MATERIAIS E MÉTODOS}

presente trabalho consiste em um estudo descritivo transversal, de natureza quantitativa, baseado nos dados dos sistemas Retratos da Atenção Primária à Saúde e Sinan, além de pesquisa bibliográfica em Galvão (2019), Domingues (2016), Cerqueira (2018), Lima (2016), Barcelos et al. (2017) e Neves et al. (2017). Realizou-se uma busca inicial nas variáveis do sistema Retratos da Atenção Primária à Saúde correspondentes ao tema da sífilis, examinando os atributos e, quando possível, correspondendo os dados com as notificações de casos da sífilis presentes no Sinan. A investigação dos dados foi feita utilizando a linguagem de programação Python ${ }^{1}$ e o ambiente computacional jupyter notebook ${ }^{2}$.

O banco de dados do PMAQ-AB utilizado pelo Retratos fornece dados sobre o terceiro ciclo do programa. Nesta fase, 5.324 municípios aderiram ao programa, de forma a possibilitar a análise de mais de 42.000 equipes (NAVI, 2020). As variáveis escolhidas para compor o cruzamento feito neste estudo dizem respeito à disponibilidade e acesso à penicilina e testes rápidos e são elas: I.11.1 - Teste rápido de sífilis sempre disponível, I.15.9.3 - Benzilpenicilina benzatina em quantidade suficiente; I.15.9.4

\footnotetext{
${ }^{1}$ Linguagem de programação de alto nível e interpretada que teve seu lançamento em 1991. Pela versatilidade e por possuir uma comunidade participativa, é amplamente usada para análise de dados.

${ }^{2}$ Ferramenta de desenvolvimento interativa que permite unir anotações, código e visualizações num mesmo arquivo. Suporta diversas linguagens, incluindo Python, e é comumente utilizada na ciência de dados.
}

- Benzilpenicilina procaína + benzilpenicilina potássica em quantidade suficiente; II.12.1.7 - Exames para o pré-natal solicitados pela sua equipe para serem realizados na rede de serviços de saúde: Sorologia para sífilis (VDRL); II.15.2 - Equipe recebe o exame VDRL das gestantes do território em tempo oportuno para intervenções necessárias; II.15.13 - É realizada na UBS a aplicação da penicilina G benzatina; III.13.7.3 - Durante o pré-natal, a usuária realizou: Exame de sífilis (VDRL).

A base do Sinan traz os casos notificados entre 2007 e 2017 para sífilis congênita e em gestantes, e no período entre 2010 e 2017 da sífilis adquirida. Para o tratamento destas informações, inicialmente fez-se a limpeza dos dados, que consiste em retirar colunas com presença de valores nulos, de valores de controle que não são relevantes para análise, bem como informações que são constantes como o sexo feminino na base que representa as gestantes. Foram excluídas também algumas colunas que possuíam correlação forte entre si a fim de evitar redundância de informações, como é o caso que ocorre na base de notificações de sífilis em gestantes entre os atributos de faixa etária e idade.

O processamento da base do Retratos inclui a etapa de filtragem de respostas entre 2016 e 2018, período de vigência do $3^{\circ}$ ciclo do PMAO. A abordagem escolhida tem o papel de evitar incluir na análise respostas fora desse intervalo e, assim, inconsistências durante o uso dos dados.

Tendo em vista a necessidade de combinar as bases das duas fontes, um agrupamento dos dados foi realizado para possibilitar este cruzamento. Os dados do sistema Retratos foram agrupados de acordo com o município de forma a contabilizar as respostas para cada variável, conforme exemplificado na Tabela 1. Por fim, os casos de sífilis, separados de acordo com suas classificações, são contabilizados por município e são adicionados ao conjunto de dados obtido anteriormente para 
que então seja calculada a correlação entre as ocorrências.

Tabela 1 - Exemplo de organização dos dados do sistema Retratos após agrupamento.

\begin{tabular}{c|c|c|c}
\hline \multirow{2}{*}{ VAR } & IBGE & \multirow{2}{*}{110001} & \multirow{2}{*}{110002} \\
\cline { 2 - 4 } & CÓDIGO & & \\
\hline \multirow{4}{*}{1.11 .1} & 1 & 3 & 10 \\
\cline { 2 - 4 } & 2 & 0 & 0 \\
\cline { 2 - 4 } & 9997 & 0 & 0 \\
\hline \multicolumn{3}{|c}{ Fonte: Autoria própria (2020). }
\end{tabular}

A correlação foi calculada utilizando a função corr() da biblioteca pandas ${ }^{3}$, cujo resultado é uma matriz que informa a correlação entre cada par de colunas do dataset, utilizando o método de Pearson ${ }^{4}$. Este método possui a característica de eliminar valores nulos antes de efetuar os cálculos, de modo que houve a necessidade de remover do conjunto as linhas dos municípios que não possuíssem resposta para alguma variável e contabilizar 0 casos para as cidades para as quais há a informação das variáveis, mas não de notificações de sífilis. Além disso, a fim de reduzir o tamanho da tabela resultante, as métricas calculadas que não relacionam os casos de sífilis e as variáveis do PMAO foram desconsideradas para esta análise.

Todos os códigos contendo as análises e os comandos que geram as imagens estão presentes no repositório no endereço https://github.com/nymarya/ analise-correlacao-sifilis.

$\bigcirc$ presente estudo foi realizado com dados secundários, disponibilizados pelo Ministério da Saúde na Internet, garantindo o anonimato dos indivíduos, em conformidade com a Resolução n. 466/2012 do Conselho Nacional de Saúde.

\footnotetext{
3 Biblioteca da linguagem Python criada para manipular e analisar dados.

${ }^{4}$ Cálculo desenvolvido por Karl Pearson que mede a correlação linear entre duas variáveis, tendo o resultado um valor entre -1 e 1.
}

\section{RESULTADOS E DISCUSSÕES}

Este estudo investigou os dados presentes no sistema Retratos da Atenção Primária à Saúde, a fim de entender as características de infraestrutura e oferta de serviços coletadas pela pesquisa do $3^{\circ}$ ciclo do PMAO. Ao verificar a matriz de correlação exibida na Figura 1, vê-se que as notificações de sífilis adquirida têm forte correlação positiva (coeficiente com valor 0.88) com a resposta 1, correspondente a "Sim" para a variável II.12.1.7, que sinaliza a solicitação de exames VDRL para pré-natal. Isto significa dizer que existe uma associação do número de casos com o acesso satisfatório e a presença do teste VDRL nos municípios. O mesmo padrão é encontrado para as notificações de casos de sífilis em gestantes e de sífilis congênita, com correlação positiva de coeficientes iguais a, respectivamente, 0.89 e 0.82 . A abordagem dos casos de sífilis durante o pré-natal é fator decisivo na qualidade de atendimento ofertado e está diretamente relacionado com desenvolvimento da Atenção Primária à Saúde no país que apesar dos diversos desafios possui importante contribuição ao sistema de saúde (SARACENI et al, 2019; ARANTES et al, 2016).

Permanecendo no tópico relativo a testes diagnósticos, houve a indicação de uma forte correlação entre os casos e o fornecimento do resultado de VDRL das gestantes em tempo hábil (variável II.15.2), com coeficientes iguais a $0.88,0.89$ e 0.81 para ocorrências de sífilis adquirida, em gestantes e congênita, respectivamente. $\bigcirc$ acontecimento foi observado também quando a comparação é feita com a realização de teste de sífilis, VDRL, durante o pré-natal por parte das usuárias do SUS (variável III.13.7.3), obtendo coeficientes iguais a 0.82, 0.86 e 0.78 para ocorrências de sífilis adquirida, em gestantes e congênita. Estes resultados também foram observados em outros estudos no Brasil (DOMINGUES et al; SARACENI et al, 
2017; BENZAKEN et al, 2019). Para a variável III.13.7.3 verifica-se que a resposta "Não se aplica", de código 998, apresenta coeficientes que indicam correlação forte com o número de notificações para as três classificações da infecção, o que pode apontar a influência exercida por usuários do sexo masculino ou que nunca engravidaram nas respostas aos questionários.

De acordo com os resultados obtidos, a realização de exames de forma responsável e eficiente nas unidades básicas de saúde está diretamente relacionada a um maior número de casos de sífilis notificados. Este efeito pode ser uma indicação de que os exames estão sendo realizados nos lugares necessários. Estudos realizados no Brasil relataram que quanto maior o número de consultas de pré-natal realizadas, maior é a chance de ter sido oferecido o teste de sífilis para as gestantes e menor a chance de transmissão vertical (SARACENI et al, 2012, SARACENI et al, 2017).
No que se refere à disponibilidade de medicamentos, é notada uma correlação forte, de natureza positiva, entre as notificações de sífilis adquirida com a sinalização de que é feita a aplicação de penicilina G benzatina (variável II.15.13, coeficiente igual a 0.90 ) e de que há a disponibilidade de benzilpenicilina benzatina em quantidade suficiente (variável I.15.9.3, coeficiente igual a 0.72 ). A realização da aplicação de penicilina $\mathrm{G}$ benzatina nas unidades básicas de saúde também está fortemente correlacionada ao número de casos de sífilis em gestantes e sífilis congênita, o que pode ser verificado por meio dos coeficientes obtidos, em ordem, 0.91 e 0.81 . Este dado provoca uma discussão da qualidade de atenção à saúde, pois uma maior número de testes na gestação leva a um maior número de notificações de sífilis na gestação, entretanto não justificaria um maior número de casos de sífilis congênita se o diagnóstico tivesse sido realizado precocemente durante o pré-natal (SARACENI et al, 2017).

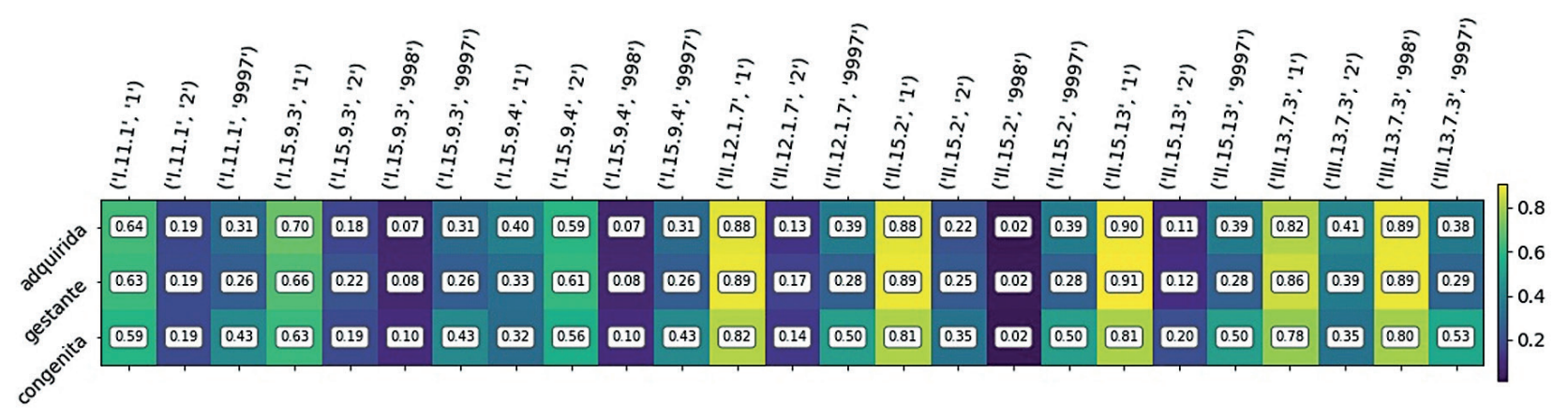

Figura 1 - Matriz de correlação entre respostas e casos notificados de sífilis adquirida, em gestante e congênita.

Fonte: Autoria própria (2020).

No entanto, para a variável I.15.9.4, que indica que a quantidade de benzilpenicilina procaína + benzilpenicilina potássica na unidade é suficiente, não é encontrada relação forte entre as respostas e a quantidade de casos notificados. Para a resposta "Sim", é diagnosticada uma correlação dita fraca, pois os coeficientes se encontram no intervalo de 0.3 a 0.5, enquanto para a resposta "Não" a correlação encontrada é chamada moderada, com coeficientes de valores entre 0.5 e 0.7 , ambas as relações de caráter positivo. A tendência para a resposta "Não" é uma possível evidência para a falta de medicamentos em áreas que possuem muitos casos da infecção. A Atenção primária enfrenta diversos desafios e precisa ser aprimorada para que as lacunas ainda existentes na rede de atenção sejam corrigidas 
para que a oferta de serviços seja feita de forma integral (ARANTES et al, 2016).

Quanto aos testes rápidos, é realizada a comparação entre o quantitativo de casos e as respostas da variável "Teste rápido de sífilis sempre disponível" de código l.11.1. Para todas as classificações da infecção foram encontradas correlações de intensidade moderada e sentido positivo, com os coeficientes 0.64 para casos de sífilis adquirida, 0.63 em gestantes e 0.56 para sífilis congênita. A divergência destes resultados para os de exames, que indicaram de modo geral uma cobertura mais satisfatória, pode evidenciar a insuficiência dos testes em lugares com alta incidência de casos de sífilis, visto que é conhecido que a amplificação da detecção de casos assintomáticos implica no aumento de notificações do agravo (FIGUEIREDO et al, 2020). Em especial, os testes rápidos são de suma importância para a identificação de casos durante o pré-natal (FIGUEIREDO et al, 2020).

Ao analisar a base de dados do Sinan, os 5 estados que apresentam o maior número de casos de sífilis notificados são São Paulo, Rio de Janeiro, Rio Grande do Sul, Minas Gerais e Paraná. Com a base do PMAQ, conforme mostrado na Figura 2, vemos que, enquanto São Paulo e Rio Grande do Sul apresentam uma boa cobertura feita com testes rápidos, obtendo para a pergunta 1.11.1, descrita como "Teste rápido de sífilis sempre disponível", uma porcentagem de respostas "Sim" acima de 88\%, no Rio de Janeiro, essa taxa chega somente a 53\%. Ademais, apenas $28.78 \%$ das equipes mineiras afirmam ter os testes sempre disponíveis, sendo este o pior índice no país para a variável, o que reforça a hipótese citada anteriormente. O Brasil apresenta diferenças regionais em vários aspectos, inclusive no acesso à saúde e perfil dos usuários (GOUVEIA et al, 2005; GUIBU et al, 2017), a situação não é diferente em relação aos casos de sífilis (SARACENI et al, 2017; BENZAKEN et al, 2019).

A utilização de dados secundários pode ser citada como uma limitação deste estudo, incluindo a possibilidade de ocorrer uma falácia ecológica quando dados agregados são analisados. Entretanto, os sistemas de informação em saúde no Brasil estão coletando progressivamente dados de melhor qualidade e completude. Portanto, a variabilidade nas estimativas é um pouco reduzida à medida que os dados ganham robustez. Essas informações podem ser compartiIhadas com gestores públicos para auxiliar no planejamento em saúde, conforme previsto na agenda estratégica do Ministério da Saúde do Brasil para eliminação da sífilis

Teste rápido de sífilis sempre disponível Minas Gerais

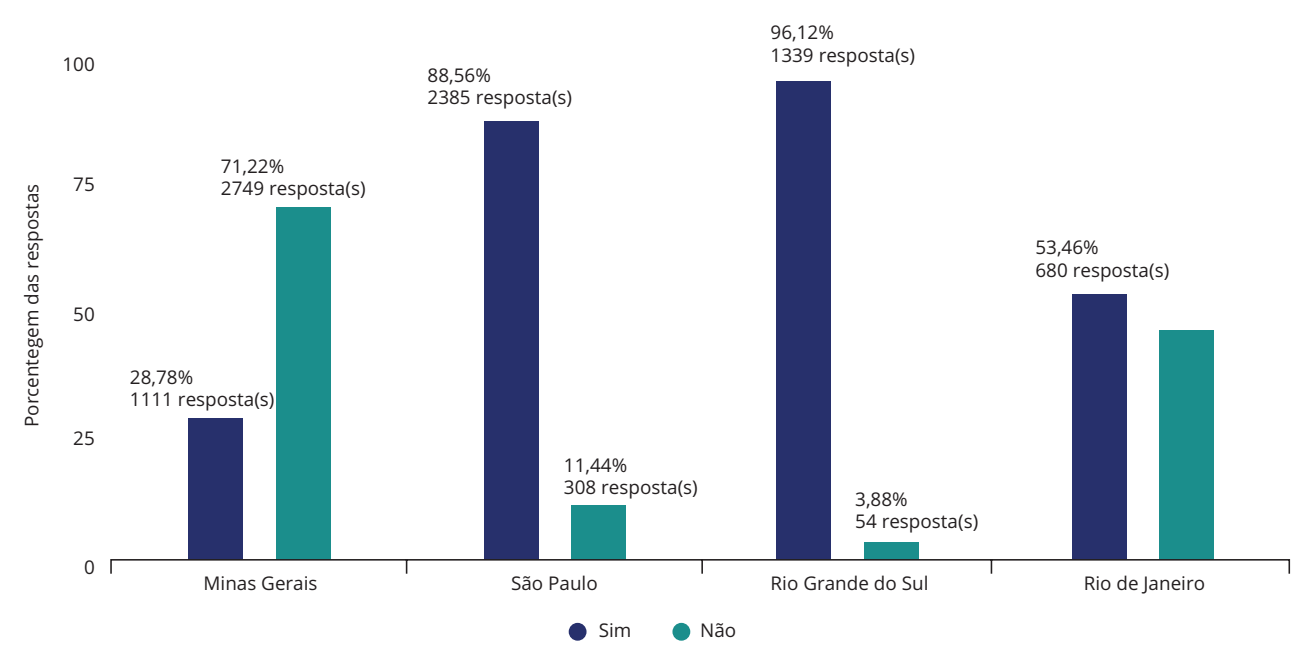

Figura 2 - Gráfico comparativo sobre a disponibilidade de teste rápidos de sífilis nos estados. Fonte: Retratos da Atenção Primária à Saúde - NAVI (2020). 


\section{CONCLUSÕES}

Para enfrentar a epidemia de sífilis no país, é preciso monitorar os casos e realizar testes diagnósticos e prover tratamento adequado na atenção primária. Neste quesito, aplicando a metodologia abordada neste estudo, verificou-se que na atenção primária do Brasil ainda não apresenta uma boa cobertura de testes rápidos e oferta de medicamentos em áreas com um número alto de casos notificados. Isso ocorre mesmo em situações onde a disponibilização e aplicação de testes diagnósticos parecem ser satisfatórias e proporcionais à demanda. $O$ primeiro resultado pode ser inferido ao interpretar as métricas que correlacionam as variáveis que indicam teste rápido de sífilis sempre disponível, quantidade suficiente de benzilpenicilina benzatina e de benzilpenicilina procaína + benzilpenicilina potássica, que abordam testes e medicamentos, com o quantitativo de notificações de sífilis. Este difere da conclusão feita sobre variáveis de oferta e aplicação de exames, levando em conta solicitação de exames de sorologia para sífilis (VDRL) para pré-natal, recebimento de exame VDRL das gestantes em tempo hábil para intervenções necessárias e realização desses exames durante pré-natal.

A escassez de estudos utilizando dados para diagnosticar a situação da sífilis no cenário nacional gera um alerta para a necessidade de realizar mais análises de modo a construir indicadores cada vez mais robustos. Para isso, é imprescindível haver mais ferramentas que promovam a transparência de dados da saúde e também fazer uso das plataformas já existentes, como o Retratos da Atenção Primária à Saúde. 


\section{REFERÊNCIAS}

ARANTES LJ, et al. The benefits and challenges of the Family Health Strategy in Brazilian Primary Health care: a literature review. Cien Saude Colet. 2016 May;21(5):1499-510. doi: 10.1590/1413-81232015215.19602015.

BARCELOS, M. R. B. et al. Qualidade do rastreamento do câncer de colo uterino no Brasil: avaliação externa do PMAO. Rev. Saúde Pública, São Paulo, v. 51, 67, 2017. Disponível em: http://www.scielo. br/scielo.php?script=sci_arttext\&pid=S0034-89102017000100261\&lng =en\&nrm=iso. Acesso em: 19 maio 2020.

BENZAKEN AS, et al. Adequacy of prenatal care, diagnosis and treatment of syphilis in pregnancy: a study with open data from Brazilian state capitals. Cad Saude Publica. 2019 Dec 20;36(1):e00057219. doi: 10.1590/0102-311X00057219.

BRASIL. Ministério da Saúde. Programa Nacional de Melhoria do Acesso e da Qualidade da Atenção Básica (PMAQ). Disponível em: https://aps.saude.gov.br/ape/pmaq, 2020.

BRASIL. Ministério da Saúde. Secretaria de Vigilância em Saúde. Boletim Epidemiológico Sífilis 2019. p. 1-44, 2019. Disponível em http://www.aids.gov.br/pt-br/pub/2019/ boletim-epidemiologico-sifilis-2019

BRASIL. Ministério da Saúde. Secretaria de Vigilância em Saúde. Departamento de Vigilância Prevenção e Controle das Infecções Sexualmente Transmissíveis, do H. e das H. V. Protocolo Clínico E Diretrizes Terapêuticas Para Prevenção Da Transmissão Vertical De Hiv, Sífilis E Hepatites Virais. Brasília: Ministério da Saúde, 2018.

BRASIL. Secretaria de Vigilância em Saúde; Secretaria de Atenção à Saúde, Ministério da Saúde. Agenda de ações estratégicas para redução da sífilis congênita no Brasil. Brasília: Ministério da Saúde; 2016. Disponível em http://www.aids.gov.br/pt-br/pub/2017/ agenda-de-acoes-estrategicas-para-reducao-da-sifilis-no-brasil

CERQUEIRA, B. G. T. de. Avaliação e melhoria da qualidade da assistência à sífilis gestacional na atenção primária à saúde. 2018. 58f. Dissertação (Mestrado Profissional em Gestão da Qualidade em Serviços de Saúde) - Centro de Ciências da Saúde, Universidade Federal do Rio Grande do Norte, Natal, 2018. 
DOMINGUES, R. M. S. M.; LEAL, M. do C. Incidência de sífilis congênita e fatores associados à transmissão vertical da sífilis: dados do estudo Nascer no Brasil. Cad. Saúde Pública, Rio de Janeiro, v. 32, n. 6, e00082415, 2016. Disponível em: http://www.scielo.br/scielo. php?script=sci_arttext\&pid=S0102-311X2016000605002\&lng=en\&nr $\mathrm{m}=$ iso. Acesso em: 21 jan. 2020.

GALVÃO, A. B. Uma ferramenta colaborativa para a análise de dados na Saúde Pública: aplicação no estudo de séries temporais para o Projeto Sífilis Não. 2019. 61f. Dissertação (Mestrado em Engenharia Elétrica e de Computação) - Centro de Tecnologia, Universidade Federal do Rio Grande do Norte, Natal, 2019.

FIGUEIREDO, D. C. M. M. de et al. Relação entre oferta de diagnóstico e tratamento da sífilis na atenção básica sobre a incidência de sífilis gestacional e congênita. Cadernos de Saúde Pública [online]. v. 36, n. 3 [Acessado 28 Janeiro 2021] , e00074519. Disponivel em: <https://doi.org/10.1590/0102-311X00074519>. ISSN 1678-4464. https://doi.org/10.1590/0102-311X00074519

GOUVEIA GC, et al. Satisfação dos usuários do sistema de saúde brasileiro: fatores associados e diferenças regionais. Cad Saude Publica. 2005;21 Suppl:109-18. doi: 10.1590/ s0102-311×2005000700012.

GUIBU IA, et al. Main characteristics of patients of primary health care services in Brazil. Rev Saude Publica. 2017 Nov 13;51(suppl 2):17s. doi: 10.11606/S1518-8787.2017051007070.

LIMA, J. G. Atributos da atenção primária nas regiões de saúde: uma análise dos dados do Programa Nacional de Melhoria do Acesso e da Qualidade da Atenção Básica. 2016. 244 f. Dissertação (Mestrado em Saúde Pública) - Escola Nacional de Saúde Pública Sergio Arouca, Fundação Oswaldo Cruz, Rio de Janeiro, 2016.

NEVES, M. et al. Primary Care Dentistry in Brazil. Journal Of Ambulatory Care Management, v. 40, p. 35-48, 2017.

NÚCLEO AVANÇADO DE INOVAÇÃO TECNOLÓGICA - NAVI. Retratos da Atenção Primária à Saúde. Disponível em: https:// retratos.navi.ifrn.edu.br. Acesso em: 18 mar. 2020.

ORGANIZAÇÃO MUNDIAL DE SAÚDE - OMS. Global health sector strategy on sexually transmitted infections, 2016-2021: Towards ending STIs. Report No.: WHO/RHR/16.09. Geneva: WHO, jun. 2016. Disponível em: https://www.who.int/reproductivehealth/publications/ rtis/ghss-stis/en/. Acesso em: 15 jan. 2020. 
SARACENI V, MIRANDA AE. Coverage by the Family Health Strategy and diagnosis of syphilis in pregnancy and congenital syphilis. Cad Saude Publica. 2012 Mar;28(3):490-6. doi: 10.1590/ s0102-311×2012000300009.

SARACENI V, et al. Epidemiological surveillance of vertical transmission of syphilis: data from six federal units in Brazil. Rev Panam Salud Publica. 2017 Jun 8;41:e44. doi: 10.26633/RPSP.2017.44.

SORTICA, A. C. Rede de atenção à saúde, sífilis e educação em saúde, a intersecção necessária: um estudo de caso sobre sífilis em gestante e congênita no município de Esteio. 2017. 122 f. Dissertação (Mestrado Profissional em Saúde) - Universidade Federal do Rio Grande do Sul, Porto Alegre, 2017. 